\title{
Computer automated procedures for assessing some experiences of family membership
}

WILLIAM H. SHERIFF, JR. NATIONAL INSTITUTE OF NEURO LOGICAL DISEASE AND STROKE, NATIONAL INSTITUTES OF HEALTH, Bethesda, Md. 20014

In a way, this was an unusual application for us. The Section on Technical Development services both the National Institute of Neurological Diseases and Stroke and the National Institute of Mental Health in the Intramural Research program at the National Institutes of Health. We went from 1962 until last year with one classical LINC, extended to $2 \mathrm{~K}$ memory after a year of operation. As we operate with the equivalent of one full-time programmer, we let the LINC out on a laissez-faire basis; if you are willing to program it, you can use it, with the Section offering subroutine support and advice. For a 3-year period, we averaged better than $17 \% / 2 \mathrm{~h}$ per week on a 7-day week using this LINC. If you take a 2-year Research Associate and give him free rein on a computer, you often enter in the morning and find a bleary-yed Research Associate. This approach has proven quite productive.

Although most of our work has been in neurophysiology and neuropsychology, I happened to get ego-involved in a problem brought to us by Dr. David Reiss of the Adult Psychiatry Branch, National Institute of Mental Health. He has been studying family interactions in schizophrenia. Schizophrenics have been studied backwards and forwards for many years, but always in terms of the relationship between a schizophrenic and the therapist or the relationship between a schizophrenic patient and the environment of the mental hospital. Dr. Reiss, along with Dr. Lyman C. Wynne and others, has decided that it would be much more fruitful to study the relationship between the schizophrenic and his family.

Dr. Reiss started this line of research at the Massachusetts Mental Center prior to coming to National Institutes of Health. His research unit is the family, containing an adolescent with a behavioral problem, studied as a group. The usual grouping is the father, mother, adolescent with a behavioral problem, and a normal adolescent sibling. NIH has a pool of such families living in the Washington, D.C., area that are willing to come in periodically, as a group, as research Ss. The $S$ has usually been a patient in the Clinical Center and is undergoing study and therapy by members of the Branch.

In past studies, Dr. Reiss has confronted these families with problem-solving tasks, usually on the order of the analogy type of problems found on intelligence tests, varying the degree of interaction (information sharing) between the members of the family. This procedure involved the $\mathrm{E}$ in the mechanical difficulties of moving screens, sorting cards, and in general, acting as a traffic director. He could not adequately observe or repeat the procedure in a standard, objective manner. He admittedly came to us reluctantly, a psychiatrist coming to a computer, but he was forced into it.

$\mathrm{He}$ approached us with a specific problem for a pilot project and a testable hypothesis. In previous studies, he had found that there seemed to be two different modes that families used for handling problems. With one method, an e nvironment-sensitive mode, the family reacts to cues from the environment and from other members of the family, weighing them with a degree of rational credibility in solving problems. Other families react in a consensus-sensitive manner. When these families are working together, they tend to ignore or distort the cues that come to them from from the environment if they conflict with the cues that they receive from other members of the family. They rely primarily on the consensus of the family. It is as if they are defending their integrity as a family by distorting the rest of the environment.

Dr. Reiss wanted to set up a pilot project to test this hypothesis. The study used eight families, four rated as consensus-sensitive and four rated as environment-sensitive. Donald F. Uggla and James S. Bryan of our section designed an interface to remotely control five Teletypes by the LINC. The experimental rooms were some distance from the computer room, and on another floor, so LINC was programmed to enable the $\mathrm{E}$ to control the experiment from one of the Teletypes. The four experimental Teletypes were set up in a soundproofed room. The E's Teletype was set up in an observation room that overlooked the experimental room through one-way mirrors. The E could see and hear the Ss; they could not see or hear the E. The Ss were seated in a row at their Teletypes in the following order: mother, $\mathrm{S}$, father, sibling. Movable screens were in the room to block the Ss from view of each other during the experimental phase of the problem.

The interface was designed to allow a character from the Teletype to be held by a buffer, one channel for each Teletype, and gated into the LINC accumulator with an OPR command, the code in bits 0.7 and the Teletype number in bits 8-10. The output data was written directly by LINC in its serial format with the appropriate channel code. The LINC was interrupted by the control logic every $9.1 \mathrm{msec}$, and the bit was transmitted via an OPR line. A single bit must be transmitted at every interrupt, except at idle conditions at logical 1. The write cycle receives top priority in the program, since the read cycle is significantly faster. We decided to use a message-in, message-out storage space in upper memory, only sending or acting on completed messages. Upper memory was also needed for one of the problem conditions, limiting us to a maximum of 32 characters per message. If I had it to do over again, I would act on individual characters received or sent rather than on messages. Even then, there would not have been enough space to control all the contingencies, such as a too-long message inserting an end-of-message flag in someone else's message area. Such things can only be controlled by good instructions and cooperative Ss with a computer this size.

We used an interrupt routine to receive and send individual characters. This routine scanned the message-out area for flags, only sending a message when completed. It placed incoming characters in the appropriate message-in area, putting a message-complete flag in when it received a carriage-return code. The main line routine scanned the message-in areas for flags, acted on the messages, evaluating and scoring, and then distributed the appropriate messages to the various message-out areas. The procedures depended to a great deal on the cooperation of the Ss, and we were very pleased to find that the Ss did cooperate beyond our expectations. The Teletype keys were modified, allowing only the letters of the alphabet and three control keys, end of message, error, and one that doesn't really matter for this presentation.

The problem was presented in three modes, explained later. The following procedure was constant for all modes. The family was given a sample trial. First, they were given instructions on the procedures. Then, they were given an example of a logical sequence, for example, a " $\mathrm{C}$ " followed by two "Xs" then a "C." They were then allowed to test different sequences on the Teletypes, using the 
Table 1

Printout from Family in Study

Example: Parents-VDMDMDMDMDMDMV; Children-VMMMMMMMVV

\begin{tabular}{lccc}
\hline \multicolumn{1}{c}{ Mother } & Subject & Father & Sibling \\
\hline VDMDMDMV+ & FCCCHH - & VFMFMFMVV - & VICJCJCVV - \\
VDMV+ & BHBHCC - & ABCD- & VDMMDDMDV- \\
VMDMDMDMV- & ERERERE- & VDMV+ & VDMDMDMDMDMV+ \\
\hline
\end{tabular}

particular presentation mode, in the family order mentioned previously. In this case, whenever they typed a " $C$ " followed by any number of "Xs" followed by a " $\mathrm{C}$," they received a " $t$ " indicating a correct response. All other responses were graded with a "-." The responses of the individuals were distributed according to the model format, and all members of the family were requested to communicate freely so that they would know the manner in which the responses were distributed.

For the experimental conditions, the families were isolated from one another by the screens and were instructed not to communicate. They were each given a printed sheet with 1 correct sequence and 10 test sequences. They were asked to evaluate the 10 test sequences with paper and pencil, turn them in, and then proceed with the Teletype routine, exactly like they did in the sample problem. After 22 trials on the Teletype, they were again given 10 examples to score by paper and pencil, to give some indication of learning during the experimental procedure.

In the sample problem, they were all given the same example. In the experimental procedure, two members were given one example and two members another, each set involving a different logical sequence. Success in the problem was determining the fact that there are two
Table 2

Printout from Family in StudyMember A (MOTHER)

EXAMPLE

PARENTS-CXXXXXXXC CHILDREN-XCCCCCCCCX

YOUR TURN

XCCCX+

YOUR TURN

CXXXXC+

$+\mathrm{XCCCCX}$

-VMMMMV

+ CXXXXXC

YOUR TURN

CXXXXXXXXXXXXXC+

$+C X C$

-VBBBBV

-WFFFFW

YOUR TURN

$\mathrm{XCCX}+$

$+\mathrm{XCX}$

$-\mathrm{ZXXXZ}$

$-\mathrm{CX}$

YOUR TURN

$\mathrm{XCCCCCCCCCCCX+}$

Table 3

Standard Mode identical in this mode. This mode is called the public mode.

The second mode, in order of information transmitted, is called the anonymous mode (see Table 2). On this mode, the $\mathrm{S}$ is informed of his tum by having "your turn" typed on his Teletype. He types his response, and it is graded and shown to him but is stored in memory and not distributed this time to the others. Thus, on the first turn, no one gets a response from any of the other members. On the next cycle through the family, as a member types his response, it is again stored in memory. But a different response from the previous cycle is distributed to each other member of the family, so that every member gets a different response from the previous cycle's responses of the

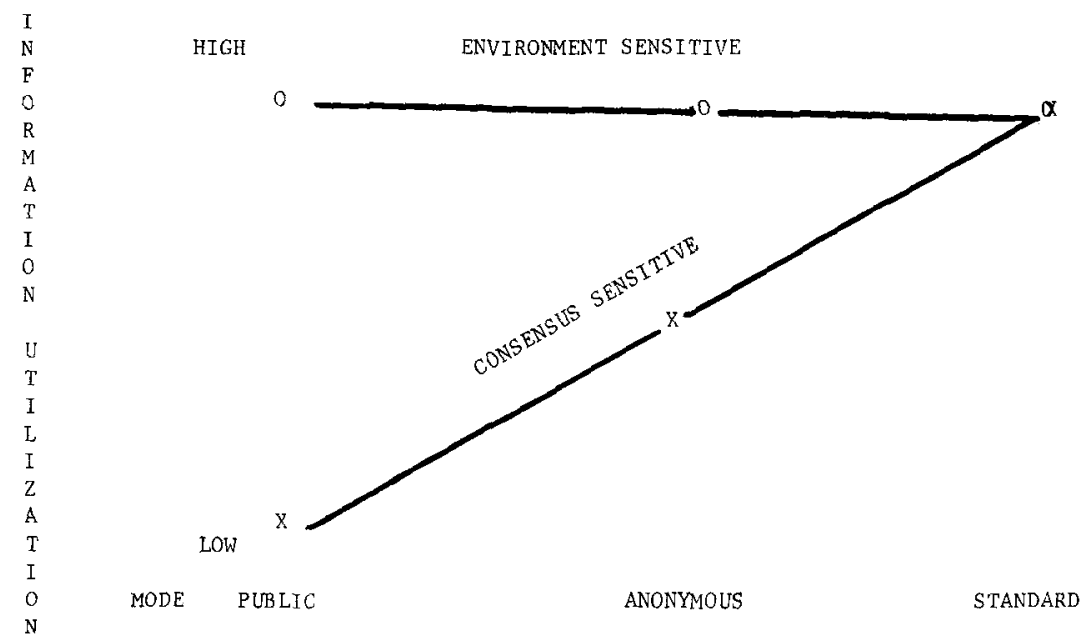

family. After the end of any cycle, every member of the family has received all of the responses made by it on the previous trial but has no way of knowing whose response he is looking at. Thus, they are all sharing the same information but do not know who transmitted the information.

The third mode, the standard mode, has minimal information shared by the family (see Table 3). In this mode, "your turn" is typed on the Teletype of the appropriate member of the family. He types his response and is given his grade. Then, responses stored in memory are distributed

Fig. 1. Predicted results. 
to the other members of the family, no member getting the same response. These responses were predetermined by the $E$ from previous frequencies of correct and incorrect responses on this type of problem. All families were given the same sequence. At no time does any member of the family have access to any information from another member.

It was predicted that the consensus-sensitive families would perform (1) poorly on the public mode, in which they would have maximal conflicting, shared evidence, (2) fair on the anonymous mode, and (3) best on the standard mode.
Environment-sensitive families should perform equally well in all modes. The predicted direction is illustrated in Figure 1. Analysis of the pre- and posttest inventories tended to confirm the hypothesis.

To conclude, I would like to read three observations made by Dr. Reiss as major advantages over noncomputer methods:

(1) Observer rating scales, long bedeviled by problems of reliability and validity, can be eliminated since all of the family's performance is scored automatically.

(2) The experimental procedure is precisely standardized and can be exactly replicated using a copy of our program tape and the same standard Teletypes and computer equipment.

(3) The presence of the human $E$ has been partially eliminated, and the prospect is good for his total unemployment.

We hope to soon eliminate the human element in this procedure, but, as it stands, it severely strains a $2 \mathrm{~K}$ memory, even with microtapes. We expect to get about 3 years' good usage out of these procedures before transferring to an anticipated larger computer system.

\section{DECUS DISCUSSION}

DR. GOFF: Can you comment on new software for the PDP-12?

RICHARD CLAYTON: The most significant initial aspects of the software of the 12 is a new operating system including assembler, editor, and various peripheral programs to handle filing and program conversion, etc. It is currently dubbed the DIAL system, or Display Interacting Assembly Language. The program is patterned in the interactive sense, very much after the LAP-6 program, which Mary Allen Wilkes distributed some 2 years ago, and the user characteristics, at least in an editing sense, are very similar. The assembly characteristics are slanted very much toward the PDP-12. Specifically, it is an assembler that handles either of the two order codes, and the format for assembly, and henceforth the format with which one writes source programs. The format for the assembler is very similar to the one commonly used throughout the DEC machines. Specifically, it has six character alphanumerics plus slash for the comment delimiter, large character $i$ as the indirect bit, and different symbol characters. This departure from the classic LINC format we felt obligated to go to in trying to bring the two machines together in a single assembly language that would allow rapid, convenient assembly of programs in either or both modes. In detail, the program allows, within core, some 180 user-defined symbols, outside of the instructions that are part of the symbol set. Beyond 180 symbols, the tape is used as the symbol area, yielding, of course, a substantial decrease in the assembly rate. As soon as one goes to mass storage for the symbol table (even if one does very clever things for symbol searches-if you have to go out to some mass storage in the form of LINC tapes or DECtape), the assembly slows down fast. To that end, an additional $4 \mathrm{~K}$ of core or disk will be usable for the symbol table. The $8 \mathrm{~K}$ version is in the program but not running at the moment. We will be running DIAL at Spring Joint for those of you who are there. The machine that is there is, in effect, a production-type machine. There will be workable versions of DIAL with the first PDP-12s that go out, which are still a couple of weeks away. That will be an evolving program, and I think we'll see quite a few changes to that in the form of additions in the next year and a half. [NOTE: The name DIAL has been changed to LAP 6-DIAL because of the similarity of many of the operating features of the editor portion of the system to those of LAP.6. Although the present name is LAP 6-DIAL, the responsibility for all development, corrections, and distribution will rest with Digital Equipment Corporation.]

SEVERO ORNSTEIN: Dick, I think that one part of the laboratory instrument meaning of the LINC was the terminal frame. What have you done to it [data terminal panel assembly]?

RICHARD CLAYTON: It existed in the form of two large plug-in units in the classic LINC. In the case of LINC-8, it consisted of about 50 unused slots mounted behind the door in the upper right-hand side of the front. What happened to that on the PDP-12? To some degree, it has gone "bye-bye," a little bit anyway. We have put a large number of options prewired into the machine itself. So a number of some of the things that went into the data terminal panel assembly, such as plotters, additional Teletypes, certain things with display and $A$ to $D$, have been prewired within the machine. This provides economy of manufacturing and allows the user to request of the manufacturer that these be supplied. Further, it makes it practical to supply them at a reasonable cost and with a minimum of labor for installation and expansion of the machine. For other kinds of options, available literally "off the shelf," there are, for example, IBM-compatible tape, things of this sort These are also offered as standard options and plug onto an $\mathrm{I} / \mathrm{O}$ bus.

The data terminal panel concept, as originally implemented in the classic LINC, allowed up to two boxes to be plugged into the machine and used some predecoded operate lines. What one ran into was a limitation in the total number of device codes, if you like, available for easy interfacing. The interface structure was extremely simple but was not a bus structure. We would now call it a radial structure in that it had several sets of ports into the machine. Once those were used up in the straightforward way, you were all over, and that was in general the way one implemented the first interfaces one designed. Beyond that, one had to make a bus structure out of one of these radial ports, so that several devices could be put 